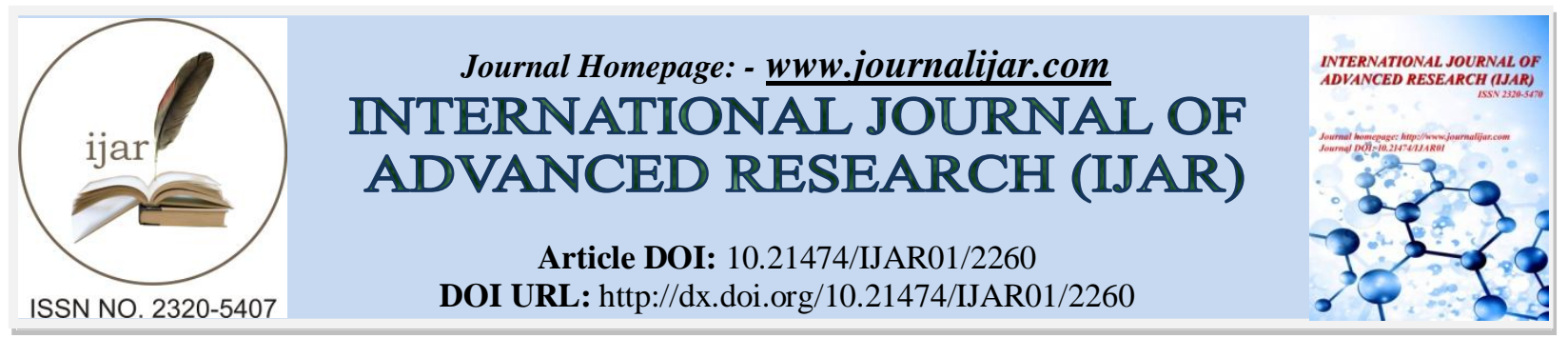

RESEARCH ARTICLE

\title{
ENHANCE BROKER TOPOLOGY FOR COMPUTATIONAL GRID SYSTEM
}

Tariq Alwada' ${ }^{1}$, Adel Hamdan ${ }^{1}$, Thair Khdour ${ }^{2}$ and Bilal Zahran ${ }^{3}$.

1. Computer Science Department, the World Islamic Science and Education University. Jordan.

2. Prince Abdulah Ben Ghazi Faculty of Information Technology, Al-Balqa Applied University. Jordan.

3. Computer Engineering Department, Al-Balqa Applied University. Jordan

\section{Manuscript Info}

Manuscript History

Received: 29 September 2016

Final Accepted: 30 October 2016

Published: November 2016

Key words:-

Distributed systems, grid computing; cloud computing; cluster computing; peer-to-peer computing; resource broker.

\section{Abstract}

Grid computing is an advanced technology that allows a collection of connected computer systems to form large, distributed system used to handle complicated scientific or business problems. The topology of brokers is a significant role to facilitate the scalability in the grid. The presented broker topologies consider both users and grid resource nodes are bound to a single broker which might increase the number of the rejected jobs. In this paper multiple brokers that work in the same domain have been introduced so that the performance of the system has been enhanced and the number of requests that the grid can process has been increased clearly.

Copy Right, IJAR, 2016,. All rights reserved.

\section{Introduction:-}

The increased reputation of the internet and the accessibility of high-speed networks and powerful computers within low-cost service components are revolutionize the way we do computing. Distributed system is performed on many systems to utilize unused distributed resource and to solve large scale problems.

\section{Distributed Systems:-}

A distributed system is a group of self-governing units that collaborate to resolve a problem that cannot be independently solved. In other words a collection of generally autonomous processors communicating across a communication network and holding the following characteristics: (Kshemkalyani et al., 2008)

$>$ No universal physical clock. This is a vital point as it establishes the key rules of "distribution" in the system.

$>$ No shared memory. This is a key characteristic that needs message exchange for communication (Risson et al., 2006).

$>$ Geographical separation. It is not essential for the processors to be on a wide-area network. Connecting processors on a Local Area Network is also being rise regarded as a small distributed system (Gupta et al., 2006).

$>$ Autonomy and heterogeneity. The processors are "loosely coupled" in that they have dissimilar speeds andeveryone can be operating various operating systems (Kshemkalyani et al., 2008).

A standard distributed system is shown in Figure 1. Each machine has a memory-processing element and the machines are connected by a communication network.

Corresponding Author:- Tariq Alwada'n.

Address:- Computer Science Department, the World Islamic Science and Education University. Jordan. 


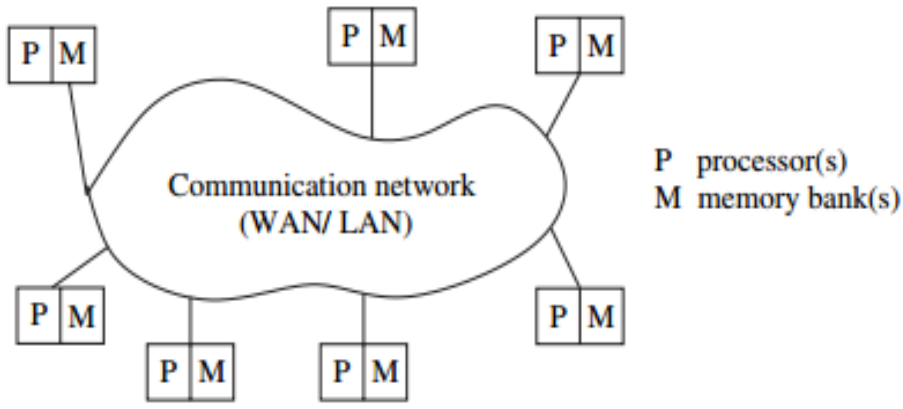

Fig.1:- "A distributed system connects processors by a communication network." (Kshemkalyani et al., 2008)

Distributed Systems can be classified into the following categories: (Kahanwal et al., 2012)

$>$ Peer-to-peer (P2P) networking has been functioning mainly on the scalability matter in distributing resources on a large number of connected processes. In a P2P structure, each node works as both a client and a server, presenting amount of the system resources.

$>$ Cluster Computing consists of a group of autonomous or stand-alone networked computers. It acts together as a single incorporated computing resource. A cluster is a local network which is made up of computers with similar hardware in the same network (What is the difference between Cloud, Grid and Cluster?, 2012).

$>$ Grid Computing is a group of machines which is owned by multiple organizations in multiple sites. The main idea behind the grid is to connect recourses together so that users can share the combined power of resources. (Kahanwal et al., 2012; What is the difference between Cloud, Grid and Cluster?, 2012).

$>$ Cloud Computing is a distributed model which supports large group of "dynamical scalable and virtual resources as a service on demand". The major rule behind cloud computing model is to present storage, computing and software as a service. Usually cloud is a set of computers owned by a single organization (What is the difference between Cloud, Grid and Cluster?, 2012; Kaur et al., 2014)

\section{Grid Computing:-}

With the improvement in communication technology and the internet, grid computing is an enhanced technology that allows a pool of connected computer systems to form a significant, distributed system used to deal with complicated scientific or business problems (Foster et al., 2004). In other words grid is a decentralized heterogeneous system that creates virtual organizations. Each virtual organization is made up of a number of different nodes. Every node can be a desktop PC, server computer, or other type of hardware, that are sharing some resources with other nodes. A major aim of grid computing is to make possible for applications to spot resources dynamically to produce distributed computing environments (Czajkowski et al., 2001).

Grid computing has appeared as a new model for distributed computing. It supports the sharing of distributed resources that may be varied in nature, and facilitates solving huge scale computing problems. With grid computing, industries can effectively employ computing and data resources and organize them for large capability workloads. The main advantage of grid computing is the capability to organize and share resources (Buyya et al., 2001; Zsolt et al., 2002).

The grid computing field includes the real networking services and connections of a possible unrestricted number of computing devices adding to the computing resources and problem resolution tasks contained the operational grid environment (Joseph et al., 2004). A grid can be owned and shared by several administrative groups which are coordinated to provide dependable, transparent, persistent computing services to a large variety of applications (Miguel et al., 2004; Karimi, 2014).

The paper is organized into sixth sections starting with an introduction followed by a brief explanation of how the grid works. The related work is discussed in Section 3. The details of proposed topology are described in section 4. Section 5 discusses the results of simulation. The paper ends with the conclusion in section 6 . 


\section{How Grid Works:-}

Grid computing is a computer network in which every computer's resources are shared among other computers in a single system (Strickland, 2008). Just as an internet user views a combined request of content using the Web, a grid user sees a single, huge virtual computer grid computing is established on an open group of standards and protocols such as Open Grid Services Architecture (OGSA) (Robbins, 2006). Memory, network bandwidth, processing power and data storage are altogether community resources that authorized users can knock into and leverage use for particular jobs (Strickland, 2008).

Resource selection is a vital matter in a grid system, where an end user and a service provider are spread geographically among many administrative domains. The grid lets completing jobs in various nodes. So as to manage job scheduling and best resource organizing at grid level, normally it is employ a Resource Broker or a meta-scheduler. A resource broker is essential in any comprehensive grid environment. The job of this broker is to dynamically recognize and distinguish the available resources, to choose and allocate the most suitable resources for a specified job and to ensure the truthfulness of the service provider (Krauter et al., 2002; Bouyer et al. 2008). Figure 2 illustrates how the grid computing system works.

These resource brokers gather information such as usage models, resource availability, hardware and software specifications, capabilities and pricing information from the individual resources, and make use of this information source in the pairing procedure between the end user and a service provider. After that the resource broker provides feedback to the end users on the available resources (Joseph et al., 2004). Furthermore, it is seeking to insert a new service by adding a layer of transparency in approaching the grid resources. Which means the end user should not be worried about the present status of a specific resource, nor should the end user concern about the process of job that been submitted (Afgan, 2004).

The Information Services and the Replica Catalogue in Figure 2 are used from the resource broker side to query about the hardware/ software and the location of the data in the grid respectively.

In this paper multiple resource brokers in the same domain have been used to match the end users requirements to the suitable resources in the grid regardless of the resource types.

\section{Related Work:-}

K Narmadha and S Thamarai Selvi in (Narmadha et al., 2007) have introduced a broker topology in which the brokers are not destined to a specific domain. The end users and resource providers have the capabilities to select the broker according to their selection. In this topology each broker is dedicated to one type of resource (compute resources consist of PCs, workstations, clusters, supercomputers, multiprocessor machines etc.). The problem of this topology is that although many brokers are presented, but each broker is responsible for only one category of resource. So if many requests come for a specific type of resource, only one resource broker is responsible for matching theses requests. In other words the other resource brokers are functionless at this stage. In addition, the topology is not really cleared nor explained in their paper.

Also K. Czajkowski and others in (Czajkowski et al., 1998) mentioned that in the process of looking for an available resource match, many resource brokers might be negotiated till available resources have been found. As soon as a resource is found, the requirements are delivered to the local management system which used job submission. But nothing has been explained about the way of using this topology in the grid system. 


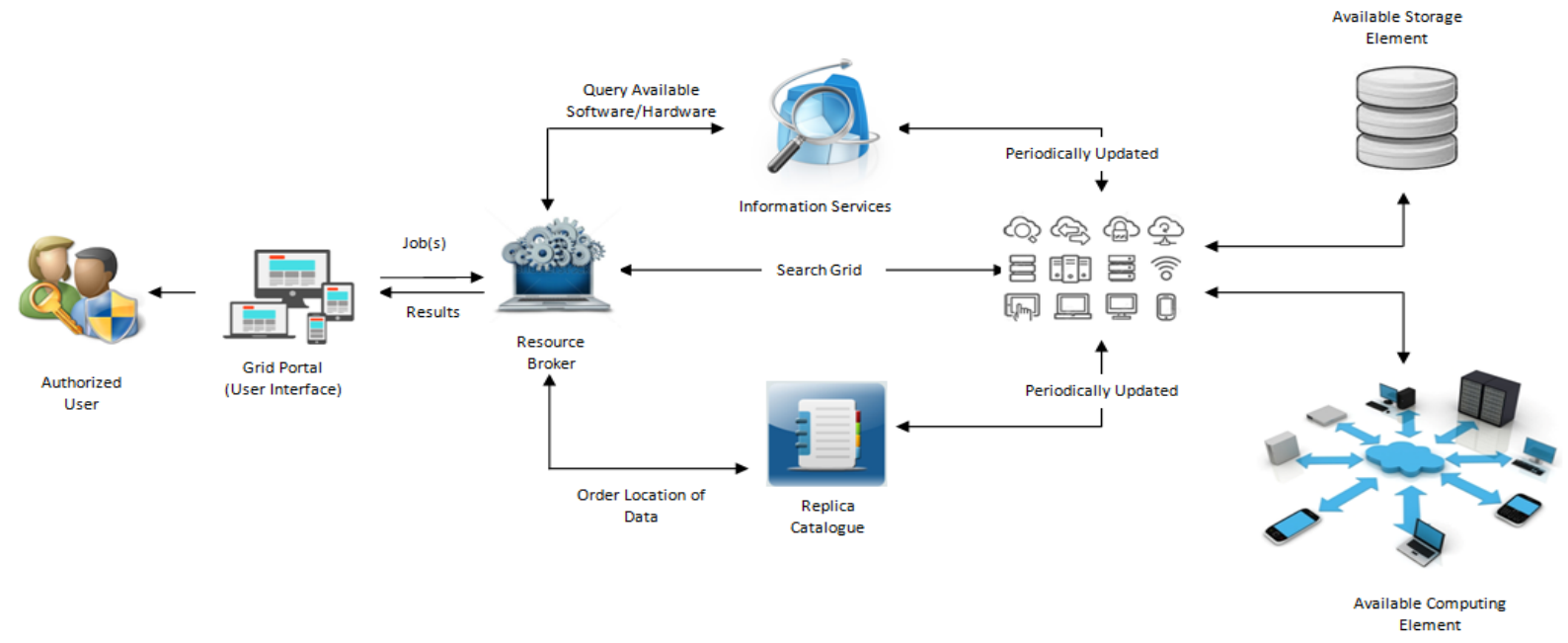

Fig.2:- Processes in Grid System (Alwada'n et al., 2014)

\section{Proposed Topology:-}

In our topology we have introduced multiple resource brokers that work in the same domain; all of them are responsible for matching the end users requirements with the available and suitable resources. Figure 3 shows this topology.

Generally, the broker is a computer or software which behaves as a conciliator among two or more parties during consultations is called as a broker. Finding out and deciding about the appropriate resources for tasks by transmitting input files of jobs to the resources, observing jobs, and returning the outputs of the job to the clients are the main functions of a broker (The Nimrod-G Grid Resource Broker and Economic, 2012).

As the authorized end user asks for a job solving, the job is sent to the grid through the grid portal. Later the available resource broker or the ones that has the least number of jobs processes the new job. Each broker is responsible for periodically reporting the number of jobs that have been handled by it and the ones with the least number of jobs is on the top of the queue as it comes first. The resource broker will locate available resources that can best match up the user's requirements and job specifications by communicating both the information service, to retrieve information about software and hardware presently free, and the replica catalogue, to detect the location of required data. Once the application has selected the suitable resources for the job, or has made advance reservations on the selected resources, the job is dispatched to those resources for execution. After that the resource broker submits the results back to the user.

\section{Simulation:-}

We have used a Jade Platform to test our job. Jade platform has the ability to provide a smooth understanding of job requests and broker responses in the case of cloud environments. Different scenarios have been conducted using this platform. Figure 4 shows part of our scenario. The figure contains two jobs which have been sent to the grid. The grid in our scenario has four brokers. In our simulation the research has been tested by applying 30 jobs in two scenarios, each with 15 jobs. In the first one; the grid contains only one resource broker. In the second scenario; the grid contains four resource brokers. A total of 30 requests have been sent to the grid in both scenarios. 


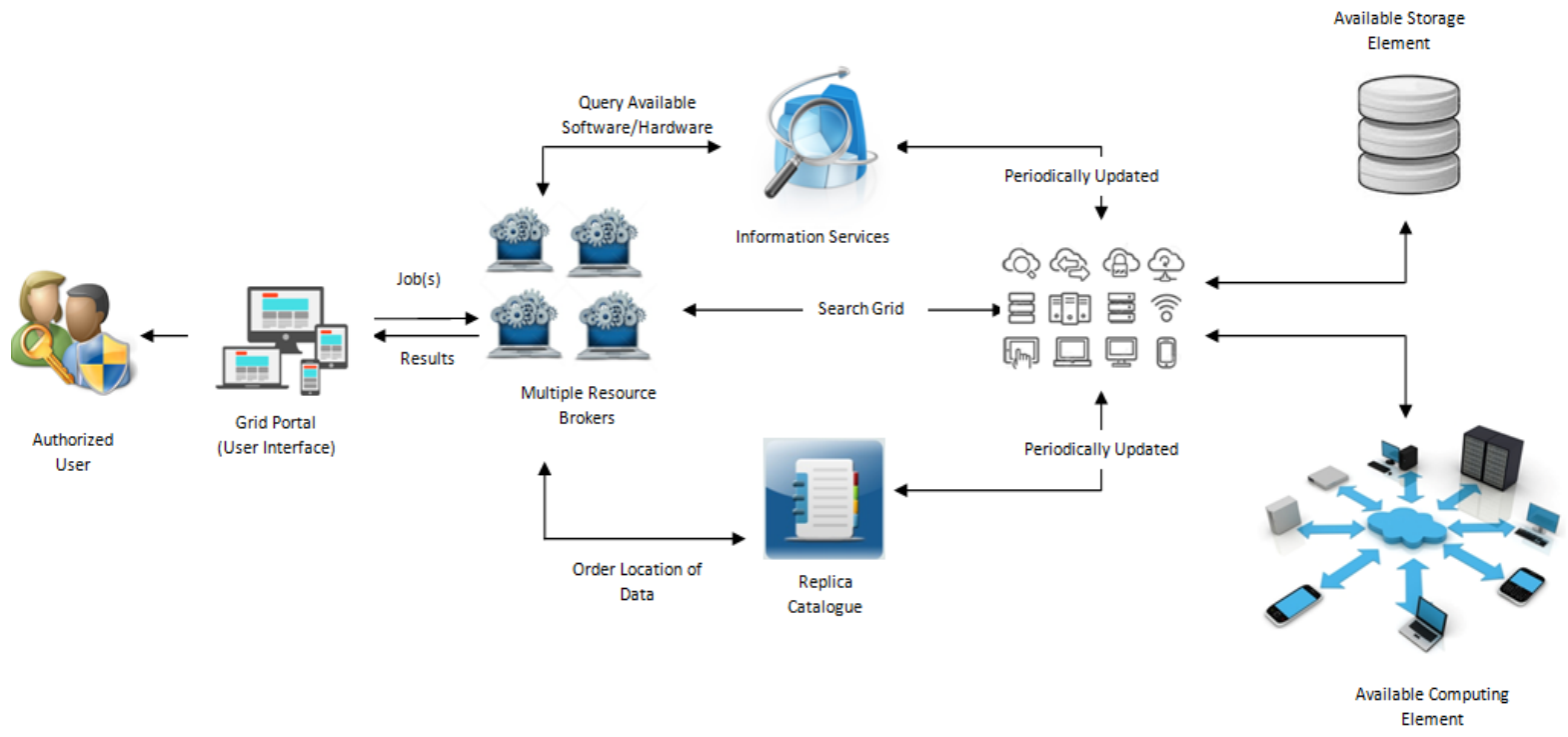

Fig.3:- Grid with Multiple Resource Brokers

As the job reaches the grid the available broker takes over to find the best match for that job. The broker contacts the Information Services and the Replica Catalogue to find the best service provider. Then it contacts the service provider directly, and finally it gets a feedback to the end user with accepting or rejecting the job.

Figure 5 shows the response time needed to fulfill the 15 requests in both scenarios. It can be noticed that when applying the four resource brokers the response time is far less than using one resource broker.

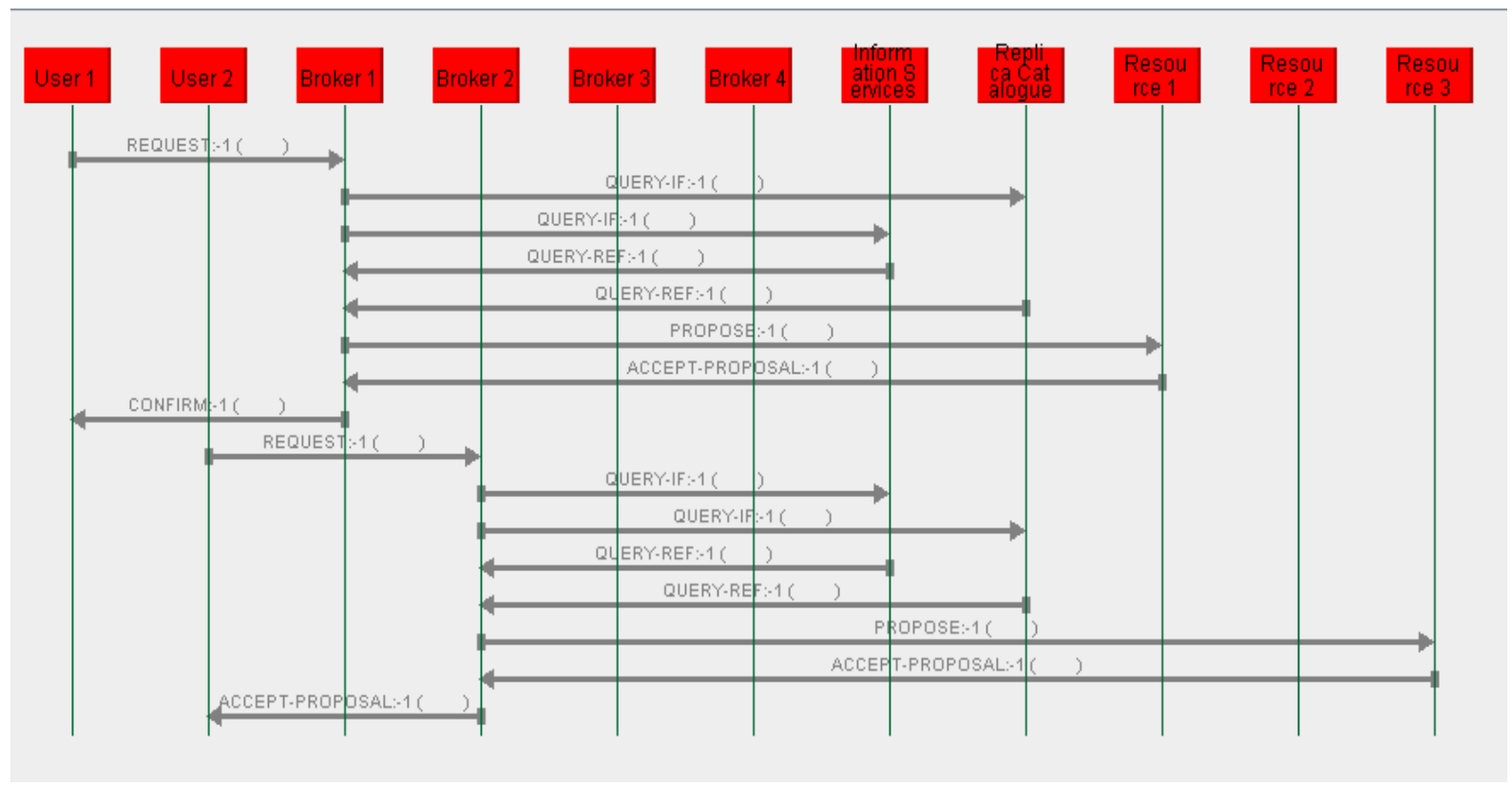

Fig 4:- Grid with four Resource Brokers. 


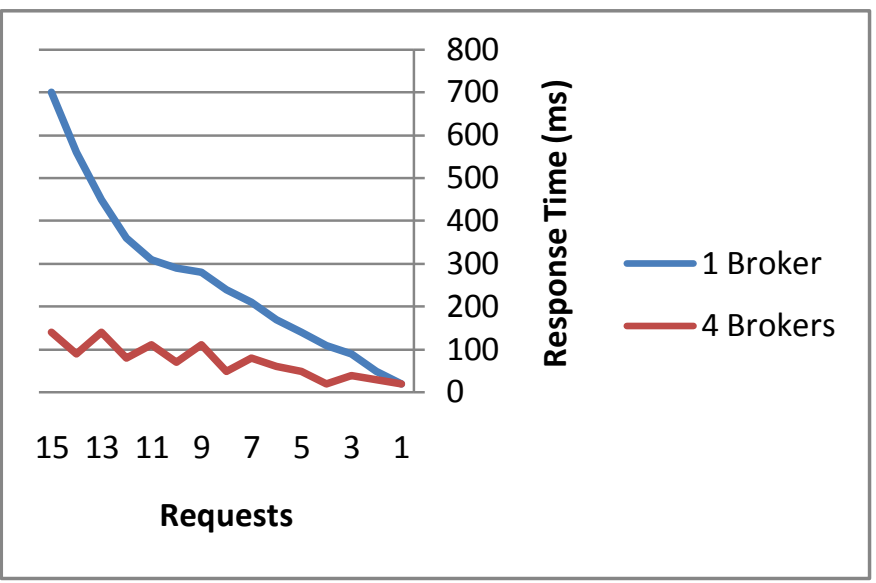

Fig.5:- The Response Time

The second tested measurement is the performance for the system. The number of requests has been calculated for five periods; 50ms, $100 \mathrm{~ms}, 150 \mathrm{~ms}, 200 \mathrm{~ms}$ and $250 \mathrm{~ms}$. According to those periods, the number of request has been calculated to show how many requests the system can handle by both cases (one resource broker against four resource brokers). Figure 6 explains these results.

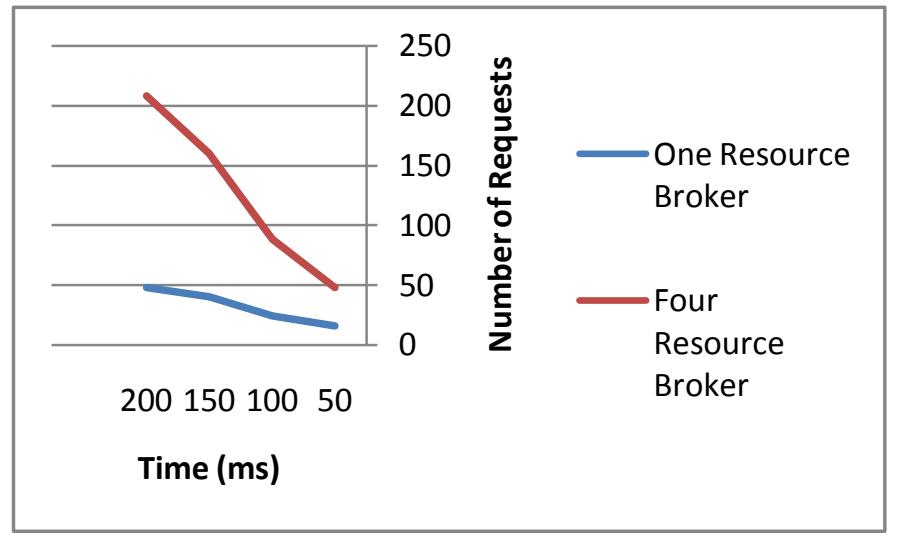

Fig.6:- Performance of the System

The last criterion that has been tested is the complexity of the system. The amount of the messages that have been exchanged in the system in single broker is less than the ones in four broker as multiple brokers work at the same time with different users and grid resources. Figure 7 shows the complexity of the system.

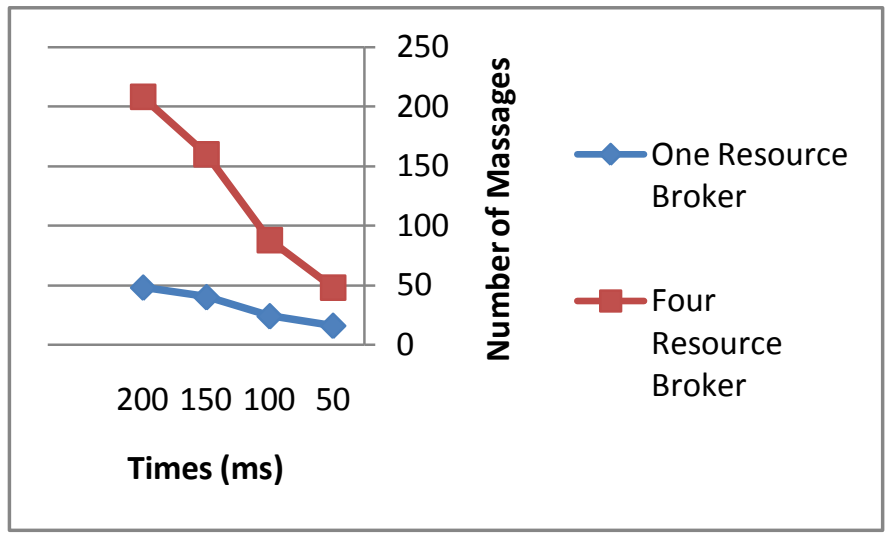

Fig. 7:- The complexity of the system. 


\section{Conclusion:-}

In this paper, a new broker topology has been introduced where multiple brokers have been inserted in the grid system instead of using one broker. The tested topology shows that the performance of the system has been enhanced and the number of requests that been processed in the grid has been increased clearly since multiple brokers are available. Although the complexity of the system has increased due of the increased numbers of communications massages between the brokers with both the users and the grid resources.

\section{References:-}

1. Afgan, E.(2004): "Role of the resource broker in the Grid". Proceedings of the 42Nd Annual Southeast Regional Conference. Huntsville, Alabama.pp 299-300.

2. Ajay, I., Kshemkalyani, D. and Singhal, M.(2008): "Distributed Computing Principles, Algorithms, and Systems". Cambridge University Press.

3. Alwada'n, T., Aldabbas, H. and Janicke, H(2014): "Mobile Grid System: Policy and Privacy". LAP LAMBERT Academic Publishing March.

4. Bouyer, A. , Karimi, M , Jalali, M. and Mdsap M.(2008): “A new Approach for Selecting Best Resources Nodes by Using Fuzzy Decision Tree in Grid Resource Broker". International Journal of Grid and Distributed Computing (IJGDC). SERSC .

5. Buyya, R., Abramson, D., and Giddy,J.(2001): "A case for economy grid architecture for service oriented grid computing”. Page 20083.1.

6. Czajkowski, K., Foster, I., Kesselman, C., Martin,S., Smith,W. and Tuecke, S.(1998): “A Resource Management Architecture for Metacomputing Systems” In Job Scheduling Strategies for Parallel Processing (pp 62 -82) Florida, USA: Springer Berlin Heidelberg.

7. Buyya.com (2012). The Nimrod-G Grid Resource Broker and Economic.Grid Broker. (pp 1-4).

8. Czajkowski,K., Fitzgerald,S., Foster, I. and Kesselman, C.(2001): "Grid information services for distributed resource sharing,". In 10th IEEE Symposium on High Performance Distributed Computing, San Francisco, California,August 7-9.

9. Foster, I. and Kesselman, C.(2004): “The Grid2: Blueprint for a New Computing Infrastructure”. Morgan Kaufmann.

10. Gupta, M., Ammar, H. and Ahamad, M.(2006): "Trade-offs between reliability and overheads in peer-to-peer reputation tracking". Computer Networks, 50(4), pp 501-522.

11. Joseph, J. and Fellenstein, C.(2004): "Grid Computing”. Prentice Hall Professional.

12. Kahanwal, B. and Pal Singh, T.(2012): "The Distributed Computing Paradigms: P2P, Grid, Cluster, Cloud, and Jungle". International Journal of Latest Research in Science and Technology Vol.1,Issue 2. pp183-187. July-August.

13. Krauter,K., Buyya,R. and Maheswaran,M.(2002): "A Taxonomy and Survey of Grid resource Management Systems", Software Practice and Experience, 32(2): 135.164.

14. Kaur, K. and Kaur Rai, A. (2014): “A Comparative Analysis: Grid, Cluster and Cloud Computing”. International Journal of Advanced Research in Computer and Communication Engineering Vol. 3, Issue 3, March.

15. Karimi, M.(2014): "Hybrid Discrete Particle Swarm Optimization for Task Scheduling in Grid Computing". International Journal of Grid Distribution Computing Vol.7, No.4.

16. Miguel,L., Bote-Lorenzo, Yannis A., Dimitriadis and Eduardo Gomez-Sanchez. (2004): "Grid Characteristics and Uses: a Grid Definition", In the Postproc. Of the first European Across Grid Conference (ACG'03), SpringerVerlag LNCS 2970, pp.219-298, Santago de Compostela, Spain, February.

17. Narmadha and, K. and Thamarai Selvi ,S. (2007): "A Topology of Specialized Brokers for Computational Grid". INTERNATIONAL JOURNAL OF IMAGING SCIENCE AND ENGINEERING (IJISE). VOL.1, NO.4, October.

18. Risson, J. and Moors, T.(2006): "Survey of research towards robust peer-to-peer networks: search methods". Computer Networks, 50(17), pp 3485-3521.

19. Robbins, S.(2006): "Lessons In Grid Computing: The System Is A Mirror". Wiley.

20. Strickland, J.( 2008): "How Grid Computing Works". Web. 06 September. http://computer.howstuffworks.com/gridcomputing.htm .

21. Stackoverflow.com (2012): "What is the difference between Cloud, Grid and Cluster?". 17 March 2012. Web. 06 September 2016. http://stackoverflow.com/questions/9723040/what-is-the-difference-between-cloud-grid-and-cluster.

22. Zsolt, N., . N'emeth and Sunderam, V.(2002): “A formal framework for defining grid systems". Page 202. 